\title{
CONCENTRACIÓN DEL CRÉDITO RURAL Y LA POBREZA EN COLOMBIA
}

Myriam Mercedes Cala Amaya

Universidade Federal de Mato Grosso

Brasil 
Panorama Económico, Vol. 27 - No. 2 (Abril - Junio de 2019), pp. 316-335

Myriam Mercedes Cala Amaya

JEL: Q13, Q14, Q18

\title{
Concentración del crédito rural y la pobreza en Colombia
}

\begin{abstract}
Resumen
Se analiza el relacionamiento entre la concentración de la pobreza a nivel departamental en Colombia y la asignación del crédito de fomento agropecuario, teniendo en cuenta los departamentos como también el tipo de tomador de crédito (pequeño, mediano y grande), a través de FINAGRO o del Banco Agrario de Colombia para el periodo 2006 - 2016. Se considera el Modelo de Análisis de Agrupamiento, jerárquico, aglomerado y por encadenamiento medio. Se analizan 19 departamentos del país cuyo Índice de Pobreza Multidimensional (IPM) se encuentra por encima de la media nacional. Además, se considera el Índice de Participación Relativa (IPR) por departamento, por línea y por usuario. Se muestra como la política de crédito de fomento en el país presenta alta concentración en departamentos típicamente receptores y se canalizan principalmente para los medianos y grandes productores. De esta manera, se concluye que los pequeños propietarios en los departamentos más alejados del centro del país reciben menores asignaciones contribuyendo a que sus niveles de pobreza perduren en el tiempo.
\end{abstract}

Palabras clave: Análisis de agrupamiento; Índice de Participación Relativa; Crédito Rural; Pobreza

\section{Concentration du crédit rural et pauvreté en Colombie}

\section{Résumé}

La relation entre la concentration de la pauvreté au niveau départemental en Colombie et l'allocation du crédit de développement agricole est analysée, en tenant compte des départements ainsi que du type d'emprunteur (petit, moyen et grand), via FINAGRO ou du Banco Agrario de Colombia pour la période 2006-2016. Le modèle d'analyse de regroupement est considéré, hiérarchique, aggloméré et par chaîne moyenne. Parmi ceux-ci, 19 départements du pays dont l'indice de pauvreté multidimensionnelle (WPI) est supérieur à la moyenne nationale sont analysés. L'indice de participation relative (DPI) est considéré par département, par ligne et par utilisateur, il montre comment la politique de crédit au développement dans le pays présente une forte concentration dans les départements typiquement récepteurs et est canalisée principalement vers les producteurs moyens et grands. De cette façon, nous pouvons conclure que les petits propriétaires terriens des départements les plus éloignés du centre du pays reçoivent moins d'allocations, contribuant ainsi à un niveau de pauvreté durable dans le temps.

Mots clés: Analyse de regroupement; Indice de participation relative; Crédit rural; La pauvreté

\section{Rural credit concentration and poverty in Colombia}

\begin{abstract}
The relationship between the concentration of poverty at the departmental level in Colombia and the allocation of the agricultural development credit is analyzed, taking into account the departments as well as the type of borrower (small, medium and large), through FINAGRO or of the Banco Agrario de Colombia for the period 2006 - 2016. The Grouping Analysis Model is considered, hierarchical, agglomerated and by medium chain. Of those, 19 departments of the country whose Multidimensional Poverty Index (WPI) is above the national average are analyzed. Relative Participation Index (IPR) is considered by department, by line and by user, it is shown how the development credit policy in the country presents a high concentration in typically receiving departments and is channeled mainly to medium and large producers. In this way, we can conclude that the small landowners in the departments furthest from the center of the country receive less allowances, contributing to their poverty levels lasting over time.
\end{abstract}

Keywords: Clustering analysis; Relative Participation Index; Rural Credit; Poverty 


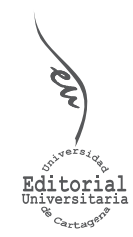

Artículo de Investigación

\section{Concentración del crédito rural y la pobreza en Colombia}

\section{INFORMACIÓN DEL ARTíCULO}

Recepción del artículo: 24/11/2018

Concepto de evaluación: 15/12/2018

Aceptación del artículo: 06/01/2019

Myriam Mercedes Cala Amaya*

Universidade Federal de Mato Grosso, Brasil

\section{INTRODUCCIÓN}

La obtención de recursos de crédito para soportar los procesos de generación de capacidad de gasto o de inversión es una condición de uso milenario, que ha tenido múltiples maneras de ser percibida a través del tiempo, desde quienes consideran la actividad de prestar y generar un lucro sobre ese monto como un ejercicio no aceptable en la comprensión de lo socialmente aceptable, y por tanto indigna tanto para quienes ofrecen el recurso como para aquellos que los requieren ${ }^{1}$, hasta los términos en que se comprende actualmente desde la perspectiva económica, donde precisamente se proponen estos apalancamientos como fundamentales en la obtención de riqueza porque es en ellos donde finalmente se puede soportar la generación de bien-estar de las comunidades.

Para un país como Colombia, que pretende ser competitivo a nivel internacional y lograr la mejora en condiciones sociales y económicas del sector rural, una de las principales estrategias que requiere atender está relacionada con favorecer el acceso al crédito especialmente encaminando acciones para el perfeccionamiento de los instrumentos financieros que resulten adecuados en términos de las particularidades regionales, con visión de fomento efectivo, donde la prioridad sea el favorecimiento de la oferta y en el que temas como tasas de interés efectivas en condiciones atractivas, cubrimiento del riesgo y asistencia técnica, sean fundamentales.

De esta manera se beneficia no solamente la seguridad alimentaria de los pueblos que cada vez se amenaza más en la medida en que la atención del sistema se canaliza a favorecer las mono producciones extensivas de dedicación prioritaria a la producción

\footnotetext{
${ }^{*}$ Autor de correspondencia: myriamcala@gmail.com ORCID: 0000-0002-8011-5427

1. Como se refleja en "El mercader de Venecia" de William Shakespeare, que refleja una visión medie-val frente al crédito.
} 
de combustibles o de materias primas que soportan explotaciones pecuarias, con destino fundamental a los mercados internacionales; sino que también, se favorece la inclusión del sector rural en la construcción de desarrollo sostenible, en la medida en que contribuye a la disminución efectiva en los niveles de pobreza, encadena procesos productivos con perspectiva de inclusión social, económica y ambiental, favorece la construcción de visión de gestión al sector en términos de disminución en la exposición al riesgo por operación, mejoramiento en los niveles de beneficio obtenidos por los dueños de los factores de producción en todos los niveles sociales, así como mejora en el aprovisionamiento de alimentos para la comunidad en general.

Todo esto procurando configurar al sector rural como un componente interrelacionado con el componente urbano de forma efectiva, provisto de infraestructura y de acceso a beneficios, que logre ser atractivo para las ciudadanos y por tanto, retenedor de comunidades, evitando así uno de los problemas más significativos en la población colombiana relacionadocon el desplazamiento a centros poblados, comoopción deacceder a beneficios históricamente concentrados tanto territorial como poblacionalmente, según donde estén los grupos de poder ( (Gutierrez, 2015); (Departamento Nacional de Planeación, 2014))

La realidad histórica colombiana deja entrever como además de buenas intenciones del legislador derivada regularmente de las presiones hechas por los grupos de productores rurales $^{2}$, de un lado, y de los grupos de poder minoritarios, que aprovechan a su favor inclusive las tímidas reformas agrarias, monetarias y fiscales en tanto tienen que ver con el componente de crédito para consolidar sus beneficios oligopólicos, de otro, no han tenido efectos reales que favorezcan la ruralidad nacional, con especial atención en los hogares de baja renta y de pequeña propiedad, que en últimas son los que soportan problemas de pobreza y pobreza extrema, sea en su calidad de residente rural o como desplazado de allí, que buscando un mejor futuro para su grupo familiar se ubica a vivir en los centros urbanos, en áreas periféricas y/o con limitadas oportunidades de participar en los beneficios derivados de la inversión social pública ( (Ospina Morales, 2014); (Kalmanovitz \& López, 2012))

A partir de los resultados obtenidos del DANE (Departamento Administrativo Nacional de Estadística, 2016, p. 823), a partir del análisis hecho sobre el III Censo Nacional Agropecuario 2014 - III CNA 2014, de la población residente en el sector rural nacional por lo menos el $45,7 \%$ está en condiciones de pobreza ${ }^{3}$, límite superior del intervalo en $84,6 \%$ alcanzado en el departamento de La Guajira y con límite inferior en $23 \%$ mostrado en el Departamento del Quindío. Debe tenerse en cuenta que el 57,5\% de los 33 departamentos están por encima de la media nacional.

En términos de tierras rurales del país, el DANE (Departamento Administrativo Nacional de Estadística, 2016, pp. 63 - 70), cuenta que a 2014, el 81,4\% se dedican primordialmente a la producción agropecuaria4, donde por lo menos el 97,8\% del tamaño de los predios se aprovechan en la actividad. El otro $18,6 \%$ se dedican a actividades no agropecuarias ${ }^{5}$

\footnotetext{
2. Mestizos, negros e indígenas, en los primeros años de la República. Luego, los campesinos que laboran tierras del estado, pequeños propietarios o que buscan redistribución de propiedad rural, hasta los 70’s del siglo XX. y aún hoy, por quienes mantienen la misma posición de lucha.

3. Teniendo en cuenta el Índice Multidimensional de Pobreza Ajustado - IMP

4. Definidas en el III CNA - 2014 como Unidades de Producción Agropecuaria - UPA.

5. Definidas en el III CNA - 2014 como Unidades de Producción No Agropecuaria - UPNA
} 
tales como transformación de productos agropecuarios, industria, comercio, minería y servicios. Sin embargo, tienen como característica importante el hecho de que por lo menos el 70,4\% de las Unidades de Producción Agropecuaria - UPA, tienen un tamaño máximo de 5 hectáreas y representan sólo el $2 \%$ de las tierras rurales ocupadas del país; mientras que el 0,2\% de las UPA, tienen por lo menos 1000 hectáreas y se distribuyen en el 73,8\% de las tierras del territorio nacional. Y aún más, el 34,5\% de las UPA totales se dedican a actividades agrícolas, que revisando el gran total nacional equivalen al $18,9 \%$ de las tierras rurales del país. El $56,6 \%$ son pecuarias, con media de cubrir el $27,7 \%$ del área rural nacional y, el 8,9\% están cubiertas con bosques naturales, y tienen una participación del 54,3\% de las hectáreas rurales nacionales, dedicadas a esta labor.

Denota esta aproximación el nivel de importancia que significa para el país social, ambiental y económicamente de los pequeños productores y de las unidades productivas en las que operan.

Anivellatinoamericanosetienen experiencias quea pesar detener escenariosterritoriales que pudieran hacerlos entender inicialmente como diferentes ${ }^{6}$, comparten con la realidad colombiana cuestiones como alta concentración de recursos, principalmente de dineros canalizados via crédito, subsidios y seguros para los grandes y medianos productores del sector rural, que ha generado inconvenientes en el comportamiento de variables socio ambientales y económicas.

Los inconvenientes parecen aparecer en la medida en que al estar canalizados principalmente a dotar de innovaciones tecnológicas de tipo mecánico, biológico, organizacionales y físico-químicas a los productores agropecuarios, que efectivamente resultan favoreciendo los beneficios de los inversionistas principalmente reflejados en la productividad de los recursos, son ahorradores fuertes en el uso de mano de obra, de manera que en sistemas sociales donde no se tienen propuestas alternativas que absorban a estas personas para laborar o se tengan mercados donde puedan colocar sus productos obtenidos con costos más altos, terminan desfavoreciendo las condiciones de vida de los pequeños productores y de las clases populares, que regularmente derivan sus ingresos por participar en actividades directa indirectamente relacionadas con el sector rural, en la medida en que variables como el empleo, ingreso efectivo familiar, acceso a la propiedad, servicios sociales, seguridad alimentaria, no son fáciles de lograr.

En el entender de los procesos de mercados, es normal que en función de lograr máximos beneficios y al operar en condiciones de mercados globalizados, se procura atender la tendencia cada vez más generalizada de obtener insumos en condiciones favorables en el contexto de mercados globales, para atender a la producción monocultura, direccionada fundamentalmente para exportación en calidad de commodities, por lo que las materias primas y los recursos en general, de carácter local que no cumplen con esos parámetros de beneficio, simplemente se someten a troca de proveedores o se mudan por otros que ofrezcan mejores beneficios (Dias Pereira, 2012, p. 16)

Al tener en cuenta este panorama, la pesquisa se propone a partir del supuesto de análisis según el cual los departamentos colombianos que logran tener un IPM a 2014, por encima de la media nacional de 45,7 como lo muestra la Tabla, coinciden con ser los

6. Como sucede con el panorama presentado por Díaz Pereira (2012, p. 16), de la economía Mato-grossense en Brasil. 
que tienen, durante la década de análisis 2006 - 2016, bajos niveles de participación en el monto de recursos de fomento asignados, especialmente canalizados para atender a los pequeños productores.

Para conversar de la concentración de recursos de crédito de fomento en el país tanto por área como por tipo de usuario en la ventana de análisis, se trabaja con datos estadísticos proporcionados por el Ministerio de Agricultura y Desarrollo Rural - MADR y propuestos en la Red de Información y Comunicación del Sector Agropecuario Colombiano AGRONET (Ministerio de Agricultura y Desarrollo Regional, 2017), operacionalizados a partir del modelo de Análisis de Agrupamiento (jerárquico, aglomerativo y por encadenamiento medio), con el cual se analiza el comportamiento en el crédito rural otorgado para pequeños, medianos y grandes productores, tanto a través del Fondo para el Financiamiento del Sector Agropecuario - FINAGRO, como con recursos propios del Banco Agrario de Colombia S.A - BAC, durante el periodo, en los 19 municipios que según el DANE (Departamento Administrativo Nacional de Estadística, 2015, p. 7), reflejan un Índice de Pobreza Multidimensional - IPM a 2014, superior a la media nacional, procurando ver la forma como precisamente se ha irrigado la región con recursos nuevos vía crédito de fomento en el periodo.

Luego, se toma el Índice de Participación Relativa - IPR ofrecido por el DANE (Departamento Administrativo Nacional de Estadística, 2015) con el propósito de analizar la forma como en los departamentos que reciben créditos vía recursos del BAC se concentran los recursos por montos asignados acorde con el tamaño del productor sea observando la cantidad de créditos entregados por año, como los valores entregados, en la década.

En términos de presentación, el documento se propone en tres secciones adicionales a la presente introducción. Inicialmente, se presenta la postura referencia de trabajo general que soporta el ejercicio de los investigadores. Luego, se propone el abordaje metodológico, que lleva al análisis de los resultados, para desembocar en la discusión general.

\section{REVISION DE LITERATURA}

Se considera pertinente dos elementos sobre la importancia de los pequeños productores: comprensión de ruralidad y de crédito rural.

\section{De la ruralidad}

Colombia se comprende un Estado Social de Derecho, a partir de la Reforma Constitucional de 1991, por lo que se entiende fundamental que ese sujeto denominado Estado tenga como eje común de acción la construcción de ambientes donde sea el pueblo quien construya la norma según su propia realidad y sea esta institución quien la ponga a disposición y en operación frente a un ciudadano que procura regirse por normatividad con sentido social. Esto porque el Estado es creado por el ciudadano, para que actúe en su nombre, para que trabaje con él mancomunadamente, para que procure como propósito fundamental el favorecimiento del mejor vivir y el mejor estar de la sociedad, basado en el cumplimiento del sentido común, en el equilibrio que da el reconocimiento de la individualidad y el respeto por la otredad, donde exista moderación en la proposición de límites que impidan el logro del bien común por encima de individual y donde se entienda que el gobierno es la figura administrativa de un Estado que representa a todos 
y cada uno de los miembros de la sociedad y, por tanto, no puede ser entendido como un agente externo e independiente en la construcción y favorecimiento del bienestar de los pueblos. Debe ser por principio, favorecedor de la sociedad, de quienes habitan los territorios (Corte Constitucional de Colombia, 1998, p. 3); (Angarita Barón, 1992, p. 3); (Villar, 2007, p. 83)).

El Estado del presente tiene, sobre esas pretensiones, la obligación de operar en este sentido, de manera que encuadre las condiciones definitorias de su ser en términos de territorio, poder soberano, población y orden jurídico, en procura de lograr el propósito de bienestar inclusivo, tanto de la presente como de las futuras generaciones.

Atendiendo lo propuesto por García (2010, p. 15), la territorialidad hace referencia a la demarcación de limites donde puede ejercer soberanía, pudiéndose extender a espacios físicos de otro Estado (en el caso de las embajadas, por ejemplo) y a elementos móviles (barcos, aviones...). A nivel nacional y para efectos e tomar decisiones de planeamiento principalmente, se ha optado por establecer dos categorías tradicionalmente: urbano y rural.

A pesar de las características físicas del país ya conversadas en términos porcentuales, tradicionalmente se ha planeado el territorio a partir de la compresión urbana, desde el entendido de ser un área donde se agrupan las edificaciones de forma contigua y agrupada en bloques, delimitadas por vías y avenidas, principalmente. Dada la cantidad de gente que se agrupa en ellas, se debe proveer con infraestructura importante que garantice condiciones de salubridad (acueducto, alcantarillado), acceso a servicios sociales (hospitales, escuelas, espacios de recreación...), soporte de servicios públicos (transporte, energía eléctrica...), soporte en condiciones de aprovisionamiento (mercados, centros de negocios...), entre otros factores. En esta categoría están las ciudades capitales y los centros poblados, definidos como cabeceras municipales alrededor de la cual se conciben los municipios del país (Departamento Nacional de Estadistica, 1996).

Esta clasificación histórica lleva a la centralización de las acciones tanto del Estado como de los grupos de poder, en la ejecución de gasto en la parte urbana, donde la evaluación con indicadores económicos tradicionales permite ver la relación de cubrimiento con mayor facilidad. Así, el sector rural, concebido de manera residual por el mismo documento soporte del DANE (1996), figura en el otro lado de la propuesta de planeación y, por tanto, termina colocándose en condiciones que demuestra la franca incapacidad de acceso a condiciones mínimas de progreso incluso, a menos que desde el componente económico signifique tener condiciones diferentes, que lleven a entender estas áreas como estratégicas para aplicar recursos y hacer presencia institucional.

En el año 2014 con la postura del Departamento Nacional de Planeación - DNP (2014, p. 5) se adopta un cambio en esa dualidad al empezar a conversar de la categoría de ruralidad en un área a partir de tres aspectos fundamentales: 1) Ruralidad dentro del sistema de ciudades, en la medida en que es ciudad aquella que: a) presenta movimiento diario de por lo menos el 10\% de la población a otro municipio; b) municipios con por lo menos 100000 habitantes en la cabecera municipal; y, c) municipios con menos de esa densidad poblacional ${ }^{7}$ pero que significan importancia estratégica a nivel regional para efectos de la prestación de servicios; 2) Densidad Poblacional, donde Colombia en el área rural

7. Densidad poblacional adoptada según la Organización para la Cooperación y el Desarrollo Económico OCDE, al definir lo rural como aquel territorio donde la cantidad de habitantes por kilómetro cuadrado es menor de 150. 
mantiene poblaciones de baja densidad con promedio de $10 \mathrm{hab} / \mathrm{km} 2$; con media que va de los 50 hab/km2 a los 100 hab/km2; y las más pobladas que tienen más de 100 hab/ km2. DANE, (2016); y, 3) Relación población urbano - rural, para lo cual se consideran rurales aquellos "que tienen cabeceras municipales con menos de 25000 habitantes y densidades poblacionales de 10 hab/km2 y 100 hab/km2". Y como Rurales Dispersos aquellos con "áreas no municipalizadas - ANM, con cabecera municipal pequeña y densidad poblacional menor a 50 hab/km2". DNP (2014, pp. 9 - 10).

En el III CNA 2014, el DANE (2016, p. 26), utiliza por primera vez la clasificación en UPA e UPNA. Las dos como opciones de explotación de la unidad de producción agropecuaria, sin importar su ubicación ${ }^{8}$ con gerencia única 9 . Sin embargo, cuando se revisa la forma como se clasifican los usuarios del crédito de fomento del sector rural, se habla aun de tipos de usuarios como pequeño, mediando y grande productor, así como de grupos poblacionales de características especiales ${ }^{10}$ como usuarios del sistema, situación que conduce a dificultades de compresión tanto para acceso a los recursos como para seguimiento en términos de efectividad de la política, en la medida en que las condiciones propuestas para unos y otros no permiten hacer convergencias fáciles entre unos y otros (Fondo para el Financiamiento del Sector Agropecuario, 2018, p. 2).

\section{Del crédito rural}

El desarrollo del ejercicio del crédito implica la generación de un contrato donde las dos partes intervinientes acuerdan una doble obligación: de quien pide prestado, de pagar $\mathrm{y}$, de quien presta, de entregar el dinero. Implica el manejo de factores importantes tales como la confianza generada, porque va a tener de vuelta el capital prestado a otra persona por un tiempo en los términos y tiempos acordados; también implica, transferencia de propiedad del recurso prestado y la otra persona puede uso de él como quiera; y, también, se soporta la generación de lucro a favor de quien presta el dinero para ser usado por quien lo requiere (Albuquerque (1995, p. 12); Almeida y Zylbersztajn (2008, p. 267))

Igual, el crédito implica tener en cuenta tres aspectos significativos: 1) las condiciones que tiene que demostrar el tomador, que garanticen al dador la seguridad de tener de vuelta su dinero; 2) el tiempo, en el que se debe cumplir, no solo en términos de la vuelta del capital sino también de los beneficios derivados del acuerdo; y, 3) un monto intereses, que se propone en el sentido de mantener el valor del capital en el tiempo y de generar beneficios para quien pudiendo tenerlo inactivo o colocado en otro negocio, decide llevarlo a las manos del tomador del crédito, quien a su vez tiene apalancamiento para desarrollar su actividad.

Sin importar la ubicación ni la actividad económica que defina su objeto, una unidad productiva requiere dinero para sus actividades que como propone Gitman (2016), puede ser provisto también de fuentes externas que van desde los dineros de familiares hasta los percibidos por opciones no tan tradicionales como el Factoring, por ejemplo.

8. Resguardo, comunidades indígenas o territorios de comunidades negras.

9. De una o varias personas naturales y/o jurídicas, sea como colectividades agropecuarias, cooperativas u organismos oficiales.

10. Mujer rural de bajos ingresos, víctimas del conflicto armado interno, desplazado e re-insertados, vinculados con programas de desarrollo alternativo, comunidades negras. 
Sin embargo, el panorama del pequeño productor colombiano que comparte condición con la mayoría de los productores rurales, radica en la mínima tasa de formalización empresarial, que le impiden tener fluidez en mercados como el financiero, comercial y hasta en la misma bolsa de valores ${ }^{11}$. Esto adicionado a la fragilidad que representa el sector derivado del alto riesgo por la dependencia de factores naturales como el clima, variación de precios y de las condiciones agronómicas que no permiten ter seguridad para los acreedores respecto de la seguridad de recibir su dinero y los beneficios pactados.

Esta realidad, al conversar con la perspectiva de flaqueza del pequeño productor frente al mercado de crédito de fomento como lo propone Wenner (2002, p. 1), podría llevar a concluir que en ese Estado Social de Derecho, las condiciones deben direccionarse entonces a proponer políticas que favorezcan a estas poblaciones que además del riesgo natural, que puede corregirse con inversión en técnicas y tecnologías de producción de avanzada, tienen dificultades relacionadas con encuadramiento en el mercado en condiciones competitivas y comparativas. De ahí, que pareciera singular la necesidad de formular política pública direccionada a favorecer las condiciones de mejora en la calidad de vida del campesino, del productor rural.

Las decisiones en este sentido tomadas por el Estado colombiano se encaminan a comprender la banca de fomento directo a través del subsidio colocado en los empréstitos donde se requiere de un agente financiero que actúa como intermediario ${ }^{12}$. Según Gutiérrez (2015, p. 1), entre 2002 - 2013 la proporción de colocaciones por las líneas de fomento sólo representan el 7,7\%, del total de crédito otorgado en el periodo:

"La capacidad que tienen los productores agrícolas y los hogares y las empresas de las zonas rurales para realizar inversiones a largo plazo, asumir riesgos y crear corrientes de ingresos estables está determinada por los productos y servicios financieros disponibles" (Gutierrez, 2015, p. 1)

Luego de la creación del Sistema Nacional de Crédito Agropecuario - SNCA, el legislador pretende unificar los criterios para administrar lo referente al financiamiento directo del sector, en la medida en que antes de sue existencia se general dificultades de eficiencia y efectividad en la labor de fomento dada la multiplicidad de instituciones que aun cuando confluyen en su obligación de ayudar al sector rural, terminan tomando decisiones que no son coherentes entre $\mathrm{si}^{13}$, especialmente en lo que tiene que ver con el manejo de procesos que favorezcan el desarrollo inclusivo y eviten la concentración de recursos en usuarios y zonas determinadas, aun quedando por fuera y de forma sistemática otros sectores. Con él, actualmente se tienen dos opciones básicas para tener recursos dedicados al financiamiento del sector agropecuario y rural con recursos de fomento en el país:

1. Fondo para el Financiamiento del Sector Agropecuario de Colombia - FINAGRO, configurado como una entidad de carácter mixto, que funciona financiando con crédito de redescuento, colocados a través de la banca privada y mixta y que procura la canalización de dineros al sector mediante líneas de redescuento. También se encarga de administrar

11. A nivel nacional se opera con la Bolsa Mercantil de Colombia - BMC

12. Conocido como banca de segundo piso.

13. Banco Cafetero, Instituto Colombiano de la Reforma Agraria - INCORA, Programa de Desarrollo Rural Integrado - DRI, Proexport, Caja de Crédito Agrario, Industrial y Minero - Caja Agraria, Asociaciones de productores y comercializadores de insumos, entre otros. 
el Fondo Agropecuario de Garantías, que es cubrir el riesgo que puede significar un crédito otorgado para un usuario frente al sector financiero prestamista, en el caso de que el deudor no cumpla con los requerimientos solicitados como soporte por la entidad y por el sistema. La idea es poder soportar solo aquellos proyectos que son técnica, financiera y ambientalmente viables, debiendo obviamente, cumplir con todas las demás exigencias propuestas.

2. Los recursos propios del Banco Agrario de Colombia S.A - BAC, que es responsable de promover el acceso al crédito por parte de los productores agropecuarios y rurales según lo define su razón de existir y que en buena parte asume las funciones que, desde su creación en la primera mitad del siglo XX, tiene la Caja Agraria.

Con esta reorganización ocurrida en las últimas décadas y propuestas a la luz de la visión propuesta por la Constitución Nacional, se esperaría que durante el periodo analizado de pudiese dar mejora en términos de desconcentración de recursos por usuario y por áreas de aplicación.

\section{METODOLOGÍA}

La propuesta se desarrolla como investigación aplicada en los términos de Crewell (2007, p. 13), porque parte de la realidad del país, denotando lo sucedido en el área específica del crédito de fomento agropecuario y rural. Desde el objetivo de la pesquisa, es de carácter descriptivo, atendiendo a Gil (2002, p. 43), en la medida en que a partir de la observación que ocurre en las estadísticas de los años 2006 - 2010 - 2014 y 2016, se nota la forma como evoluciona el financiamiento rural colombiano en términos de describir el comportamiento y la concentración, en términos del monto asignado por departamento. Los resultados de la investigación se expresan por el abordaje cuantitativo (Crewell, 2007), a partir de informaciones de naturaleza monetaria, con datos consolidados de financiamiento rural en Colombia, que son dispuestos por el Ministerio de Agricultura y Desarrollo Rural - MADR, de dominio público, a través del sitio electrónico Red de Información y Comunicación del Sector Agropecuario de Colombia - AGRONET, en planillas electrónicas, donde se encuentran disponibles montos de crédito contratado por tipo de línea y por usuario. A partir de allí, se organizan de acuerdo a los requerimientos del proceso investigativo, se hace el tratamiento, se infiere y se hacen las interpretaciones en función de atender los propósitos del ejercicio.

Para analizar la existencia de concentración en los montos asignados como financiamiento rural en el país, se seleccionan $19^{14}$ de los 33 departamentos que, atendiendo los datos ofrecidos por el DANE (2016, p. 823), tienen un IPM superior a la media nacional observada para 2014 de 45,7, como se referencia en el Cuadro 1. 
Tabla 1.

. Índice de Pobreza Multidimensional Ajustado - IPM 2014 Área Rural

\begin{tabular}{|c|c|c|c|c|c|}
\hline Ordem & Departamento & IPM & Ordem & Departamento & IPM \\
\hline 1 & Guajira & 84,6 & 20 & Nariño & 44,9 \\
\hline 2 & Vichada & 80,6 & 21 & Putumayo & 42,9 \\
\hline 3 & Guainía & 75,9 & 22 & Meta & 42,3 \\
\hline 4 & Vaupés & 75,2 & 23 & Huila & 41,0 \\
\hline 5 & Magdalena & 67,6 & 24 & Antioquia & 40,9 \\
\hline 6 & Cesar & 66,1 & 25 & Santander & 36,3 \\
\hline 7 & Chocó & 65,9 & 26 & Valle del Cauca & 34,2 \\
\hline 8 & Bolívar & 62,7 & 27 & Caldas & 33,8 \\
\hline 9 & Amazonas & 59,7 & 28 & Boyacá & 33,5 \\
\hline 10 & Sucre & 59,6 & 29 & Risaralda & 32,8 \\
\hline 11 & Córdoba & 58,3 & 30 & Bogotá D.C. & 26,7 \\
\hline 12 & N. de Santander & 56,8 & 31 & Cundinamarca & 26,2 \\
\hline 13 & Guaviare & 53,9 & 32 & ASPSC* & 23,7 \\
\hline 14 & Caquetá & 52,1 & 33 & Quindío & 23,0 \\
\hline 15 & Cauca & 51,6 & & Media Nacional & 45,7 \\
\hline 16 & Atlántico & 50,3 & & & \\
\hline 17 & Arauca & 50,3 & & & \\
\hline 18 & Casanare & 46,8 & & & \\
\hline 19 & Tolima & 46,1 & & & \\
\hline
\end{tabular}

Fuente: DANE (2016, p. 827) y DANE (2015).

*ASAPSC: Archipiélago de San Andrés, Providencia y Santa Catalina.

Una vez obtenida la información ofrecida en las estadísticas ofrecidas tanto por el MADR en AGRONET como por el DANE, se trabaja con el Modelo de Análisis de Agrupamiento, atendiendo el vecino más próximo, para lo cual se trabaja con el editor de Excel 2013 y con el software estadístico SPSS $23^{15}$, por medio del criterio del vecino más próximo, donde la semejanza es testada a partir de la verificación de la distancia entre los componentes más cercanos, caracterizando la distancia entre grupos con distancias más o menos relevantes (Hair Jr., Black, Babin, \& Anderson, 2009, p. 429).

Con base en esta técnica es posible inferir cuales departamentos poseen características próximas en la contratación de crédito de fomento rural, evidenciando o no la existencia de concentración. Esto teniendo en cuenta un nivel de confianza del 99\%, que permite identificar los departamentos con comportamientos más homogéneos en términos de recepción de estos recursos.

El Análisis de Agrupamiento es multivariado y, para el estudio se usa el Análisis de Clúster o de Aglomeración, por ser un abordaje usado desde hace tiempo y aceptado en ambientes de análisis espacial, gestión empresarial, competitividad, entre otros ramos de la economía. Con él se jerarquizan los datos en una estructura con forma de árbol, tipo dendograma donde, según Vieira Vasconcelos (2016, p. 26), se evalúa la semejanza entre las variables testadas.

En la práctica, los grupos especiales de usuarios del crédito se incluyen en los pequeños propietarios con quienes comparte buena parte de condiciones, de manera que para el

15. Statistical Package for the Social Science. 
estudio se trabajan como un solo bloque. Ellos junto a los medianos y grandes productores, se analizan accediendo a recursos tanto de FINAGRO como del BAC.

Para facilitar el ejercicio durante la década se toman cuatro años para comparar la aglomeración por departamentos: 2006 - 2010 - 2014 - 2016. Atendiendo a que los tres primeros años marcados significan los intervalos de periodos presidenciales, en el 2014 se realiza el III CNA, que entrega información importante sobre el particular. Sólo el 2016 está fuera del criterio, pero es el fin de la década de análisis.

Se aplica igualmente el Índice de Participación Relativa - IPR, que según Santana, Nogueira, et al (2009, p. 6) y Crocco (2003, p. 6), busca referir la relación que existe entre los montos de crédito asignados por tipo de productor, frente al total nacional asignado para el periodo.

Se maneja el indicador desde dos perspectivas: una, para revisar la evolución en la participación total de cada departamento i del Grupo en la asignación de recursos totales para el grupo en el año $j$.

$$
I P R=\left(\frac{E i j}{E j}\right)
$$

Otra perspectiva es atendiendo el monto para financiamiento rural para el productor tipo $i$ en el año $j$, respecto del total de los recursos concentrados en el país o en el grupo de análisis, durante el año $j$. Se calcula la concentración atendiendo el monto de recursos entregados por cada tipo de productor por año. Así:

IPR GRUPO: denota el monto total asignado en el Grupo al tipo de productor $i$ en el año j, respecto del total asignado para el Grupo en el año j.

IPR TG/TN: denota el monto total asignado en el Grupo al tipo de productor $i$ en el año $j$, respecto del total asignado al tipo de productor a nivel nacional en el año $j$.

IPR TG/ATN: denota el monto total asignado en el Grupo al tipo de productor $i$ en el año $j$, respecto del total asignado como crédito de fomento rural para pequeños, medianos y grandes productores a nivel nacional en el año $j$.

\section{RESULTADOS}

\section{Agrupamiento de los departamentos de alto ipm rural a 2014}

Según los resultados del DANE (2016, p. 827), el IPM para la población residente en el área rural por departamento muestra que la mejor condición la ofrece el Quindío $(23,0)$ y la más desfavorable se ubica en La Guajira (84.6).

Al hacer el Análisis de Agrupamiento para los 19 departamentos, con un nivel de confianza del $99 \%{ }^{16}$, se encuentra que Guainía, Vaupés, Amazonas, Chocó, Guaviare y

16. Como muestra la línea roja (resaltada por los autores) del dendograma. 
Guajira y Vichada, al igual que Arauca, Sucre y Caquetá, comparten la característica de no ser sujetos de asignación en montos para el Grande Productor en 2006 y 2010, cambiando esta condición paulatinamente entre 2014 y 2016, principalmente cargados a la Línea FINAGRO, así como tener asignaciones de recursos para Pequeños por debajo del grupo y de la nacional; sin embargo, estos tres últimos aunque comparten esta condición aparecen en un clúster diferente porque tienen dos características fundamentales: reciben valores por las dos líneas y para todos los tipos de productores, de mayor significancia frente a los demás del grupo y, de manera particular, se nota que los asignados para los Medianos Productores por FINAGRO y BAC, se maneja incluso con valores superiores a la media del grupo analizado y de la media nacional por departamento.

Figura 1. Comportamiento por Clúster del Grupo de departamentos. Total Anual por Tipo de Usuario. Colombia. 2006 - 2010 - 2014 - 2016. Precios corrientes (Millones de pesos)

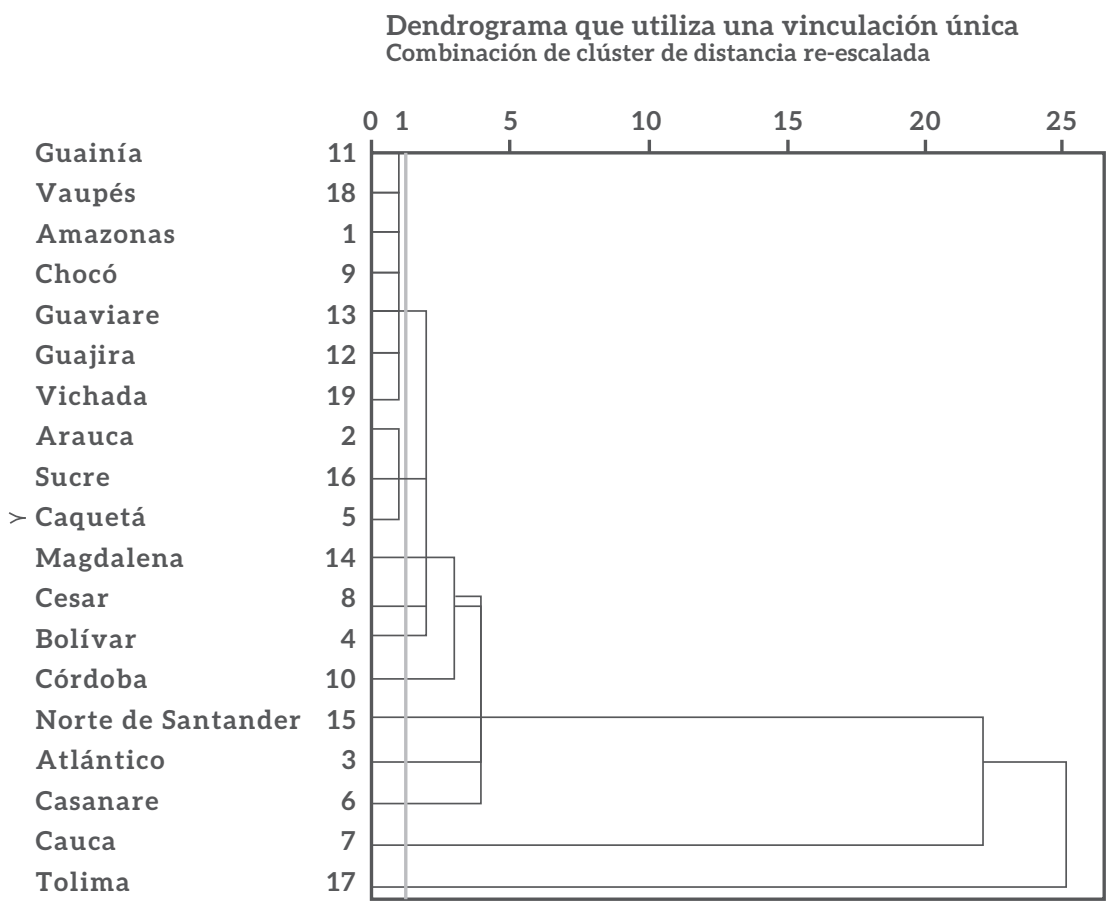

Fuente: Tomado de cálculos obtenidos en SPSS 23, a partir de datos de AGRONET, MADR (2017)

Para ver el comportamiento de los departamentos que tienen particularidades se ofrecen la Figura 2, que conversa de Clústeres en 2006 y 2010 y la Figura 3, que muestra lo ocurrido en los Clústeres en 2014 y 2016.

En el año 2006, la condición de concentración es la inexistencia de recursos Línea BAC para Grande Productores, así como el acceso de los Pequeños Productores en recursos 
de fomento en medias bien inferiores a las del grupo de estudio. Sin embargo, Sucre, Atlántico y Bolívar encuentran dineros en FINAGRO para los Grandes Productores, en montos superiores a la media de los 19 más pobres, e incluso, arriba de la nacional.

En el 2010, el clúster de bajos niveles de asignación de recursos general deja a dos entes territoriales fuera (Vichada y Arauca), pero invita a Magdalena, que con Sucre y Caquetá forma el clúster 2, recibiendo los más altos montos de dinero por las dos líneas para Pequeños (sin superar la media del grupo), pero que en Medianos supera tanto la media del grupo como la nacional y tienen asignaciones FINAGRO para Grandes.

Figura 2. Comportamiento por Clúster del Grupo de departamentos. Crédito Rural. Colombia. 2006 - 2010. Precios corrientes (Millones de pesos)

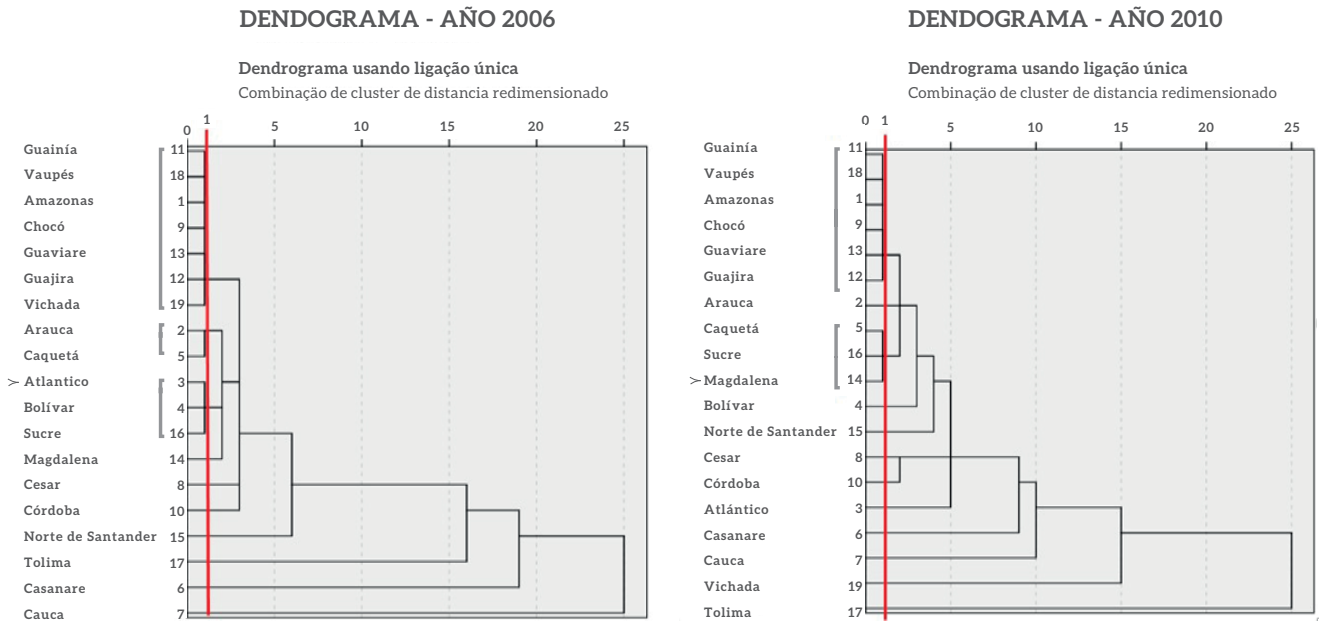

Fuente: Cala Amaya, Myriam M. (2018). Crédito de fomento agropecuario, agricultura familiar e interpretación del Desarrollo en el Departamento del Meta, Colombia, 2007 - 2014. UFMT, Brasil. 2018. *e.a.

Tasa de interés efectiva anual

Para 2014, quedan compartiendo clúster con los 7 departamentos con valores bastante alejados de la media en recursos de fomento por crédito. Guajira es quien percibe más beneficio en el año, incluyendo alguna mínima irrigación de dineros para Medianos y Grandes. En 2016, sigue la condición, sólo que ingresa nuevamente Bolívar y Sucre, quienes sólo comparten con los demás, el hecho de tener mayor acceso a recursos por la vía FINAGRO en todos los usuarios, y en recibir dineros para Grandes en media inferior a la del grupo. Vale aclarar que este clúster, en términos de comportamiento de montos para los productores se encuentra más cerca a la media nacional o del grupo, mientras que el otro clúster, está del otro extremo.

Se muestra como, aun habiendo percibido dineros en la modalidad de Grande Productor Línea FINAGRO en los años 2014 (Chocó, Guajira y Vichada) y 2016 (Chocó, Guainía, Guaviare y Vichada), los montos son bajos respecto de la media asignada para el resto del país. 
Figura 3. Comportamiento por Cluster del Grupo de departamentos. Crédito Rural.

Colombia. 2014 - 2016. Precios corrientes (Millones de pesos)
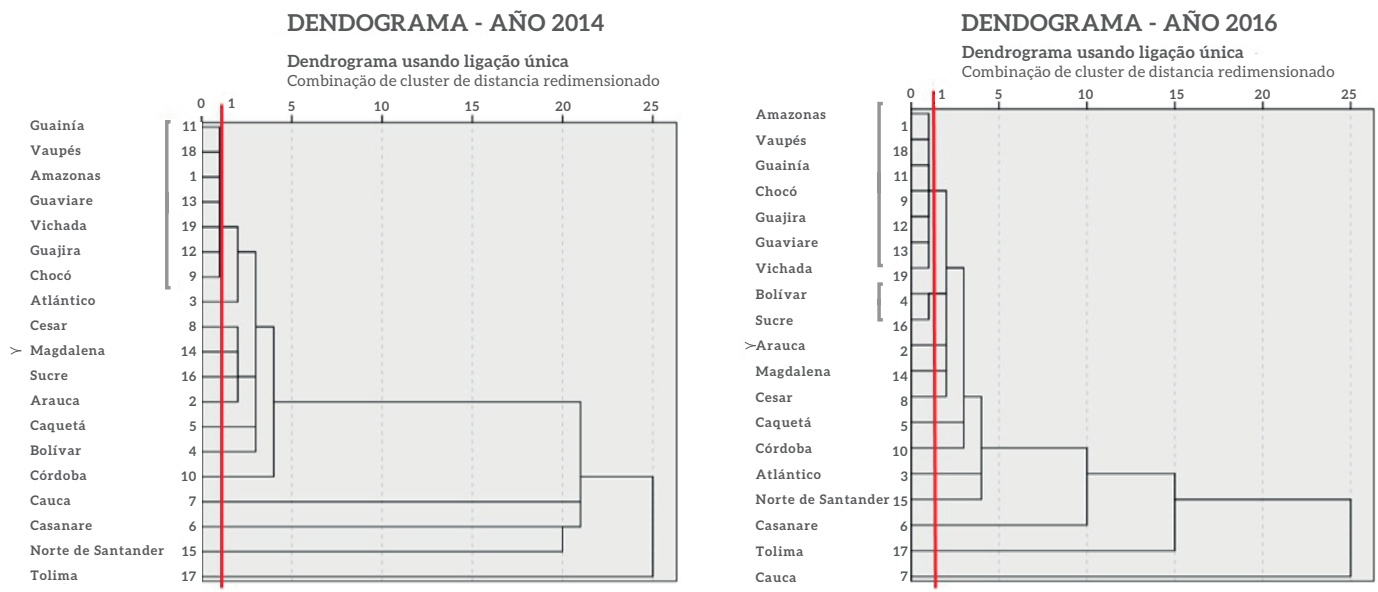

Fuente: Cala Amaya, Myriam M. (2018). Crédito de fomento agropecuario, agricultura familiar e interpretación del Desarrollo en el Departamento del Meta, Colombia, 2007 - 2014. UFMT, Brasil. 2018.

*. e.a. Tasa de interés efectiva anual

\section{Índice de participación relativa}

La canalización de los recursos de crédito de fomento para el sector rural, se presenta con altas tasas de concentración en departamentos como Tolima, Cauca, Casanare y Córdoba, que en 2006 perciben el 50,17\% y una década después aceptan el 56,61\%, mientras que Amazonas, Chocó, Guainía, Guajira, Guaviare, Vaupés y Vichada, pasan de recibir el 4,03\% al 2,90\% en el mismo periodo. Figura 4.

Figura 4. IPR por departamento y por año del Grupo amiento de 19 departamentos. Crédito Rural. Colombia. 2014 - 2016. Precios corrientes (Millones de pesos)

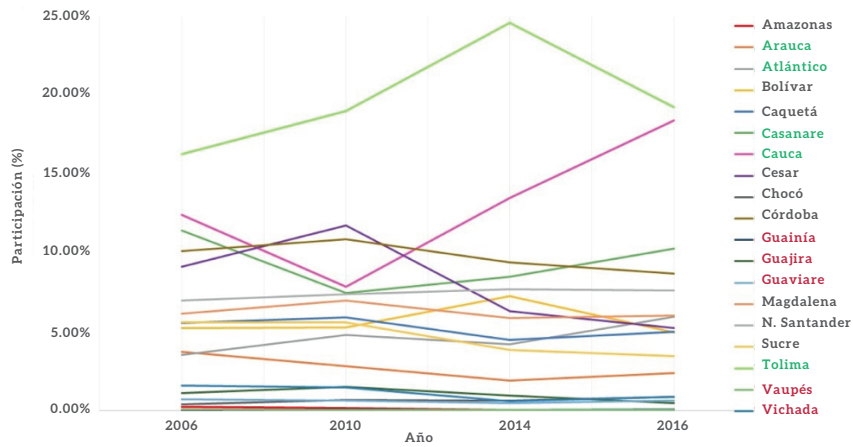

Fuente: Cala Amaya, Myriam M. (2018). Crédito de fomento agropecuario, agricultura familiar e interpretación del Desarrollo en el Departamento del Meta, Colombia, 2007 - 2014. UFMT, Brasil. 2018. Nombres en rojo ${ }^{* *}$, con IPM mas alto y en verde ${ }^{* * *}$, los que siendo pobres, tienen el IPM mas cercano a la media nacional. 
El IPR GRUPO, muestra como tomando solo al Grupo, los grandes volúmenes de crédito de fomento se canalizan para los medianos productores en los años 2006 y 2010, a donde se acude principalmente por el Crédito FINAGRO. Ya para el 2014 se canalizan principalmente para atender recursos de los Grandes Productores, siguiendo con la misma línea de preferencia.

Para los pequeños productores en la década se nota un cambio respecto de la participación en la asignación de recursos: se pasa de recibir el 28,35\% en 2006, por las dos líneas, con concentración del 55,8\% en los Medianos, a tener el 34,9\% en 2016, con el 35,94\% en manos de los Grandes productores.

Según el IPR TG / TN Del total de dineros asignados por usuario a nivel nacional, en los departamentos del Grupo se concentran en los años 2006 y 2010, los recursos de la línea FINAGRO, mientras que en los años 2014 y 2016 se usan más en estos departamentos los del BAC. Las más altas participaciones están en los dos primeros periodos por los Medianos y luego es para los Grandes Productores.

Todo esto se hace coherente con la revisión del IPR TG /ATN, que muestra como en 2006 el 12,21\% de los recursos de fomento del país se colocan vía FINAGRO para los medianos productores y para el 2010 significan el 13,5\%, mientras que para los pequeños es de 4,43\% menor aun de lo que llega para los Grandes (4,81\%).

Ya para el 2014, la concentración se da en recursos para los Grandes por FINAGRO (9,44\% del total de recursos de fomento del país de esta línea), mientras que para los pequeños llega el 10,11\% tanto por BAC como por FINAGRO. En 2016 se repite el comportamiento pues los primeros reciben el 9,97\% de la línea, mientras que los segundos perciben el $10.12 \%$ por las dos líneas. Aquí los Medianos pierden significativamente su soporte en estas regiones: pasan del 8,83\% del total nacional en las dos líneas en 2014 a ser del 8,46\% en el 2016. Luego de haber significado el 17,30\% en 2007 y el 20,74\% en 2010.

Tabla 2 .

Índice de Participación Relativa entre el grupo, en línea y por usuario a nivel nacional y según usuario a nivel nacional (2006, 2010, 2014, 2016).

(Precios corrientes)

\begin{tabular}{clc|c|c|c|c|c|c}
\hline \multirow{2}{*}{ Año } & IPR & $\begin{array}{c}\text { PP } \\
\text { FINAGR }\end{array}$ & PP BAC & $\begin{array}{c}\text { MP } \\
\text { FINAGR }\end{array}$ & MP BAC & $\begin{array}{c}\text { GP } \\
\text { FINAGRO }\end{array}$ & GP BAC \\
\hline \multirow{2}{*}{2006} & IPR GRUPO & $14.32 \%$ & $14.03 \%$ & $39.43 \%$ & $16.45 \%$ & $15.52 \%$ & $0.26 \%$ \\
& IPR TG / TN & $24.66 \%$ & $24.96 \%$ & $37.76 \%$ & $35.87 \%$ & $28.06 \%$ & $8.45 \%$ \\
& IPR TG / ATN & $4.43 \%$ & $4.35 \%$ & $12.21 \%$ & $5.10 \%$ & $4.81 \%$ & $0.08 \%$ \\
\hline \multirow{2}{*}{2010} & IPR GRUPO & $14.71 \%$ & $12.32 \%$ & $37.86 \%$ & $20.29 \%$ & $14.50 \%$ & $0.32 \%$ \\
& IPR TG / TN & $28.34 \%$ & $26.63 \%$ & $43.06 \%$ & $44.59 \%$ & $30.60 \%$ & $22.48 \%$ \\
& IPR TG / ATN & $5.25 \%$ & $4.39 \%$ & $13.50 \%$ & $7.24 \%$ & $5.17 \%$ & $0.11 \%$ \\
\hline \multirow{2}{*}{2014} & IPR GRUPO & $17.82 \%$ & $17.48 \%$ & $22.37 \%$ & $8.45 \%$ & $32.97 \%$ & $0.90 \%$ \\
& IPR TG / TN & $33.47 \%$ & $33.75 \%$ & $39.71 \%$ & $40.66 \%$ & $20.14 \%$ & $27.30 \%$ \\
& IPR TG / ATN & $5.10 \%$ & $5.01 \%$ & $6.41 \%$ & $2.42 \%$ & $9.44 \%$ & $0.26 \%$ \\
\hline \multirow{2}{*}{2016} & IPR GRUPO & $18.00 \%$ & $16.90 \%$ & $21.68 \%$ & $7.48 \%$ & $34.38 \%$ & $1.56 \%$ \\
& IPR TG / TN & $34.04 \%$ & $34.63 \%$ & $39.41 \%$ & $43.61 \%$ & $20.41 \%$ & $58.47 \%$ \\
& IPR TG / ATN & $5.22 \%$ & $4.90 \%$ & $6.29 \%$ & $2.17 \%$ & $9.97 \%$ & $0.45 \%$
\end{tabular}

Fuente: Cala Amaya, Myriam M. (2018). Crédito de fomento agropecuario, agricultura familiar e interpretación del Desarrollo en el Departamento del Meta, Colombia, 2007 - 2014. UFMT, Brasil. 2018. Valores resaltados para mostrar por año la concentración del indicador por tipo de productor. PP: Pequeño Productor; MP: Mediano productor; y, GP: Gran productor. 


\section{DISCUSIÓN Y CONCLUSIONES}

La posición del Estado Social de Derecho que se manifiesta en la Constitución de la República de Colombia no se refleja como un componente que se favorezca a través de la aplicación de la política pública de crédito de fomento, situación que como se muestra en el Cuadro 1, está buscando favorecer la concentración en áreas determinadas y en poblaciones con mayores recursos. Esto básicamente por tres razones:

En primer lugar, se supone que la política distributiva y redistributiva deben tener como propósito el favorecimiento del acceso de los ciudadanos a mejores condiciones que se reflejen en mejor calidad de vida, de manera que si en el país se tiene como factor singular de preocupación la alta concentración de predios de pequeños productores, altamente generadores de soporte en seguridad alimentaria a nivel nacional, como se explica que en toda una década se canalicen los dineros a medianos y a grandes productores, ubicados en cinco departamentos de tradicional concentración de riqueza y de producción extensiva?

En segundo lugar, si se tiene establecido por ley la protección del intermediario financiero frente al riesgo a través del Fondo de Garantías Agropecuarias - FAG y se dan condiciones especiales de atención para los pequeños productores, estos son cubiertos tanto por recursos FINAGRO como por dineros propios del BAC, a través de su infraestructura, de manera que el Estado no logra incluir a los grupos económicos y, especialmente, al sector financiero o banca privada en la atención a esta población. De manera que asume solo todo el peso de la integración de los más pobres al sistema, cuando se supone que la solidaridad es un principio fundamental de la condición de Social de Derecho.

En tercer lugar, se supone que debe existir condición de igualdad de los usuarios ante la ley y al revisar las condiciones en que opera el proceso de acceso a recursos se notan dos cosas fundamentales: de un lado, las tasas están direccionadas para que los pequeños solamente integrados con los grandes puedan salir adelante e incluso, se proponen condiciones más favorables para aquellos que decidan llevar en el negocio a población con menor capacidad económica, aspecto que favorece la concentración del poder en quienes tienen el dinero; y, de otro lado, en términos de requisitos para acceder son claramente menos favorables cuando se trate de pequeños productores, frente a lo que sucede con los medianos y los grandes.

De la forma en que está establecida la política de crédito de fomento al sector rural colombiano (por líneas y por usuarios clasificados como pequeños, grandes y medianos) y la manera como se comprenden en los diferentes escenarios dificulta no solo la evaluación y el seguimiento de la política, sino también el mismo acceso de los usuarios del común. Esto porque las comprensiones ya descritas no se encajan en aspectos como UPNA e UPA, que es de las que habla el III CNA de 2014 y que parece, se olvida en todo lo que significa la política de crédito rural, de manera que pueda hacerse apoyos que respondan efectivamente a las condiciones de las actividades a las que se canalice.

De la forma como se estructura la asignación de recursos para el crédito de fomento agropecuario en el país durante la década se notan sus limitantes en el sentido de direccionarse como un componente que efectivamente favorezca la redistribución y que se convierta en un elemento de apalancamiento efectivo en la generación de condiciones que puedan favorecer la disminución de la pobreza en el país, en la medida en que la concentración por departamentos es evidente, así como por usuarios de alto 
poder adquisitivo, dejando al gran grupo de población pobre, de bajos recursos, sin apoyo efectivo para por lo menos, seguir ocupando los espacios rurales donde hoy habita.

\section{REFERENCIAS BIBLIOGRAFICAS}

Albuquerque, M. P. (1995). Crédito rural (1 ed.). Mato Grosso: EdUFMT.

Almeida, L. F., \& Zylbersztajn, D. (2008). Crédito agrícola no Brasil: Uma perspectiva institucional sobre a evolução dos contratos. Revista Eletronica de negocios Internacionais, 3(2), 267 -287.

Angarita Barón, C. (1992). Sentencia T-406. Sentencia, Corte Constitucional de Colômbia, Bogotá. Recuperado de http://www.corteconstitucional. gov.co/relatoria/1992/t-406-92.htm

Corte Constitucional de Colombia. (1998). Sentencia SU-747/98. Estado de DerechoObjeto/Estado constitucional de derecho-Objeto. Corte Constitucional de Colombia, Bogotá. Recuperado de http://www.corteconstitucional. gov.co/relatoria/1998/SU747-98.htm

Crewell, J. W. (2007). Projeto de pesquisa. Métodos qualitativo, quantitativo e misto (2 ed.). Porto Alegre: ART MED Editoria S.A.

Crocco, M. A. (2003). Metodologia de indetificação de arranjos produtivos locais. UFMG/ CEDEPLAR, Belo Horizonte. Recuperado de http://www.cedeplar. ufmg.br/pesquisas/td/TD\%20191.pdf

Departamento Administrativo Nacional de Estadística. (2015). Colombia - Medición Pobreza Multidimensional - IPM 2014. DANE, Bogotá. Recuperado de https://formularios.dane.gov.co/Anda_4_1/index.phpcatalog/MICRODATOS

Departamento Administrativo Nacional de Estadística. (2016). Resultados. III Censo Nacional Agropecuario. Departamento Administrativo Nacional de Estadística - DANE, Bogotá. Recuperado de https://www.dane.gov.co/ files/images/foros/foro-de-entrega-de-resultados-y-cierre-3-censonacional-agropecuario/CNATomo2- Resultados.pdf

Departamento Nacional de Estadística - DANE. (2016). Metodología General III Censo Nacional Agropecuario. DANE, Bogotá. Recuperado de http://www.dane.gov.co/files/investigaciones/fichas/agropecuario/ metodologia_CNA-01_V1.pdf

Departamento Nacional de Estadistica. (1996). Conceptos básicos. Bogotá.: DANE. Recuperado de https://www.dane.gov.co/files/inf_geo/4Ge_Conceptos Basicos.pdf 
Departamento Nacional de Planeación - DNP. (2014). Definición de Categorías de Ruralidad. DNP, Bogota. Recuperado de https://colaboracion.dnp. gov.co/CDT/Estudios\%2 Econmicos/2015ago6\%20Documento\%20de\% 20Ruralidad\%20-\%20DDRS-MTC.pdf

Departamento Nacional de Planeación. (2014). Sistema Nacional de Crédito Agropecuario: propuesta de reforma. Departamento Nacional de Planeación - DNP, Bogotá. Recuperado de https://colaboracion.dnp.gov.co/CDT/ Agriculturapecuarioforestal\%20y\%20pesca/Sistema\%20Cr\%C3\%A9 dito\%20Agropecuario.pdf

Dias Pereira, B. (2012). Agropecuária de Mato Grosso: velhas questões de uma nova economía. Cuiabá: EdUFMT.

Fondo para el Financiamiento del Sector Agropecuario. (2018). Manual de sericios financiero. (FINAGRO, Ed.) Bogota. Obtenido de https://www.finagro.com. co/manualserv/index.html

García Ruiz, J. L. (2010). Introducción al derecho constitucional. Cadiz: Universidad de Cadiz. Recuperado de https://www.studocu.com/es/document/universidad -de-cadiz/derecho-constitucional/resumenes/resumen-libro-del-docentejose-luis-garcia-ruiz/485835/view?auth=0\&auth_prem=0\&prem_doc $=0$ \&new_title $=0 \&$ has_flashcards $=0$

Gil, A. C. (2002). Como elaborar projetos de pesquisa (4 ed.). São Paulo: Atlas S.A.

Gitman, L. (2016). Principios de administración financiera (14 ed.). Mexico: Pearson.

Gutierrez, E. e. (2015). Mayores oportunidades de financiamiento rural en Colombia. Banco Muncial - BM, Práctica Global de Finanzas y Mercados. Región de América Latina y el Caribe. Washington: Grupo Banco Mundial. Recuperado de http://documentos.bancomundial.org/curated/es/ 102471467999687402/pdf/AUS10747-REVISED-PUBLIC-SPANISHcolombia-spanish-web-feb9.pdf

Hair Jr., J., Black, W., Babin, B., \& Anderson, R. \&. (2009). Análise multivariada de dados (6 ed.). Porto Alegre: Bookman.

Kalmanovitz, S., \& López, E. (2012). La agricultura en el siglo XX. Bogotá: Aguilar Altea. Obtenido de https://www.researchgate.net/profile/Salomon_ Kalmanovitz/publication/5007496_LA_AGRICULTURA_EN_COLOMBIA_ ENTRE_1950_Y_2000/links/5578aa9f08aeacff20028794.pdf

Ministerio de Agricultura y Desarrollo Regional. (2017). Agronet. Recuperado http://www.agronet.gov.co/estadistica/Paginas/default.aspx 
Ospina Morales, D. S. (2014). Transformación y configuración del agro colombiano. Antecedentes del paro agrario. Revista Divergencia(16), 34 - 42. Obtenido de http://revistas.uexternado.edu.co/index.php/diver/article/download/ $3736 / 3880$

Santana, A. C., Nogueira, A. K., G., C., Sergio, \& Mendez, F. T. (2009). Identificação e caracterização do APL de Madeira e Mobiliário Nos Estados do Pará e Amapá. SOBER. 47 Congresso. 23 - 30 de outubro de 2009 (págs. 1 -19). Porto Alegre, Brasil: Sociedade Brasileira de Economia, Administração e Sociologia Rural. Recuperado de http://www.sober.org.br/palestra/13/60.pdf

Vieira Vasconcelos, V. (2016). Análise de agrupamento. Cluster Analysis. Obtenido de Análise de Agrupamentos e Regionalização: https://pt.slideshare.net/ vitor_vasconcelos/anlise-de-agrupamentos-e-regionalizao?from_ action=save

Villar, L. (2007). Estado de Derecho y Estado Social de Derecho. Revista Derecho del Estado(20), 74 - 96. Obtenido de https://dialnet.unirioja.es/descarga/ articulo/3400539.pdf.

Wenner, M. (2002). Estrategia de financiamiento rural. Banco Interamericano de Desarrollo, Washington, D.C. Recuperado de http://www.iadb.org/wmsfiles/ products/publications/documents/1481919.pdf 